\title{
Analytical and Multishaped Solitary Wave Solutions for Extended Reduced Ostrovsky Equation
}

\author{
Ben-gong Zhang \\ School of Mathematical and Computer Science, Wuhan Textile University, Wuhan 430200, China \\ Correspondence should be addressed to Ben-gong Zhang; benyan1219@126.com \\ Received 5 June 2013; Accepted 25 July 2013 \\ Academic Editor: Santanu Saha Ray
}

Copyright (C) 2013 Ben-gong Zhang. This is an open access article distributed under the Creative Commons Attribution License, which permits unrestricted use, distribution, and reproduction in any medium, provided the original work is properly cited.

We present the analytical and multishaped solitary wave solutions for extended reduced Ostrovsky equation (EX-ROE). The exact solitary (traveling) wave solutions are expressed by three types of functions which are hyperbolic function solution, trigonometric function solution, and rational solution. These results generalized the previous results. Multishape solitary wave solutions such as loop-shaped, cusp-shaped, and hump-shaped can be obtained as well when the special values of the parameters are taken. The $\left(G^{\prime} / G\right)$-expansion method presents a wide applicability for handling nonlinear partial differential equations.

\section{Introduction}

The well-known Ostrovsky equation [1]

$$
\left(u_{t}+c_{0} u_{x}+\alpha u u_{x}+\beta u_{x x x}\right)_{x}=\gamma u,
$$

where $c_{0}$ is the velocity of dispersiveness linear waves, $\alpha$ is the nonlinear coefficient, and $\beta$ and $\gamma$ are dispersion coefficients, is a model for weakly nonlinear surface and internal waves in a rotating ocean.

For long waves, for which high-frequency dispersion is negligible, $\beta=0$, and (1) becomes the so-called reduced Ostrovsky equation (ROE) [2]

$$
\left(u_{t}+c_{0} u_{x}+\alpha u u_{x}\right)_{x}=\gamma u .
$$

Parkes [3] has studied (2) and found its periodic and solitary traveling wave solutions.

In fact, by applying the following transformation [4]:

$$
u \rightarrow \frac{u}{\alpha}, \quad t \longrightarrow \frac{t}{\sqrt{|\gamma|}}, \quad x \longrightarrow \frac{\left(x+c_{0} t\right)}{\sqrt{|\gamma|}}
$$

to (2), we obtain the ROE in the neat form

$$
\frac{\partial}{\partial x} \mathfrak{D} u+\delta u=0,
$$

where $\mathfrak{D}:=\frac{\partial}{\partial t}+u \frac{\partial}{\partial x}, \quad \delta=\frac{\gamma}{|\gamma|}= \pm 1$.
Just as it mentioned in [5] and the reference therein, when $\delta=$ $-1,(4)$ is referred to the Ostrovsky-Hunter equation (OHE). When $\delta=1,(4)$ is referred to the Vakhnenko equation (VE), which is in order to model the propagation of waves in a relaxing medium $[6,7]$. Parkes [3] pointed out that (4) is invariant under the transformation

$$
u \longrightarrow-u, \quad t \longrightarrow-t, \quad \delta \longrightarrow-\delta \text {, }
$$

so that the solutions of the OHE and VE are related in a simple way.

The purpose of this paper is to study the extended reduced Ostrovsky equation (EX-ROE):

$$
\frac{\partial}{\partial x}\left(\mathfrak{D}^{2} u+\frac{1}{2} p u^{2}+\beta u\right)+q \mathfrak{D} u=0,
$$

where $\mathfrak{D}$ is defined previous, $p, q$, and $\beta$ are arbitrary nonzero constants. It is originally derived by Morrison and Parkes [8] which dubbed it as modified generalized Vakhnenko equation (mGVE) when $p=2 q$. They found that not only does it have loop soliton solutions, hump-like and cusp-like soliton solutions, but it also has $N$-soliton solutions. 
In order to investigate mGVE's $N$-soliton solution, Morrison and Parkes [8] considered a Hirota-Satsuma-type shallow water wave equation [9] of the form

$U_{X X T}+p U U_{T}-q U_{X} \int_{X}^{\infty} U_{T}\left(X^{\prime}, T\right) d X^{\prime}+\beta U_{T}+q U_{X}=0$,

where $p \neq 0, q \neq 0$, and $\beta$ is arbitrary constant. By using the transformation

$$
\begin{gathered}
x=T+\int_{-\infty}^{X} U_{T}\left(X^{\prime}, T\right) \mathrm{d} X^{\prime}+x_{0}, \\
t=X, \quad u(x, t)=U(X, T),
\end{gathered}
$$

where $x_{0}$ is a constant, (7) yields (6). So (6) and (7) are equivalent to each other under the transformation (8). Specifically, in (7), when $p=2 q$ and $\beta=-1$, it was discussed by Ablowitz et al. [10] and was shown to be integrable by inverse scatting method. When $p=q$ and $\beta=-1$, it was discussed by Hirota and Satsuma [11] and was shown to be integrable using Hirota's bilinear technique. In [12], the authors referred to (6) with $p=q=1$ and $\beta$ an arbitrary nonzero constant as the generalized Vakhnenko equation (GVE). In fact, when $p=q$ and $\beta=0$, (6) can be written as

$$
\left(\frac{\partial u}{\partial x}+\mathfrak{D}\right)\left(\frac{\partial}{\partial x} \mathfrak{D} u+p u\right)=0
$$

Clearly, solutions of the ROE are also solutions of (9) with $p= \pm 1$. So for arbitrary $p, q$, and $\beta$, if we obtain the solutions of EX-ROE, then we can also obtain the solutions of VE, GVE, mGVE, ROE, and OHE by taking the special values of $p, q$, and $\beta$.

The EX-ROE has been studied by several researchers. For example, Liu et al. [13] used Jacobi elliptic function method to obtain exact double periodic wave solutions and solitary wave solutions. Parkes [4] constructed periodic and solitary wave solutions of EX-ROE and gave the categorization of the solutions. Xie and Cai [14] used the bifurcation method of dynamic systems and simulation method of differential equations to get exact compacton and generalized kink wave solutions of EX-ROE. Stepanyants [15] applied the qualitative theory of differential equations to give a full classification of its solutions.

Recently, there are many methods being proposed to study the traveling wave solutions of nonlinear partial differential equations which are derived from physics, for example, [16-27]. As well as these methods, there are still many other methods; we cannot list all of them. Here we will use modified $\left(G^{\prime} / G\right)$-expansion method to investigate EX-ROE. As a result, three types of traveling wave solutions are were obtained. When the special values of the parameters are taken, they are reduced to some previous results which obtained by an other method.

The rest of the paper is organized as follows. In Section 2, we present a methodology of the modified $\left(G^{\prime} / G\right)$-expansion method. In Section 3, we apply the method to the extended reduced Ostrovsky equation. In Section 4, some conclusions are given.

\section{Description of the Modified $\left(G^{\prime} / G\right)$-Expansion Method}

The $\left(G^{\prime} / G\right)$-expansion method is first proposed by Wang et al. [28]. The useful $\left(G^{\prime} / G\right)$-expansion method is then widely used by many authors [29-32]. Then it is modified in [33-35]. The main steps are as follows.

Suppose that a nonlinear equation is given by

$$
P_{1}\left(u, u_{t}, u_{x}, u_{t t}, u_{x t}, u_{x x}, \ldots\right)=0 \text {, }
$$

where $u=u(x, t)$ is an unknown function and $P$ is a polynomial in $u=u(x, t)$ and its partial derivatives, in which the highest-order derivatives and nonlinear terms are involved. In the following we give the main steps of the $\left(G^{\prime} / G\right)$ expansion method.

Step 1. The traveling wave variable $u(x, t)=u(\xi), \xi=x-c t$, where $c$ is a constant, permits us to reduce (10) to an ODE for $u=u(\xi)$ in the form

$$
P_{2}\left(u,-c u^{\prime}, u^{\prime}, c^{2} u^{\prime \prime},-c u^{\prime \prime}, u^{\prime \prime}, \ldots\right)=0 .
$$

Step 2. Suppose that the solution of (10) can be expressed by a polynomial in $\left(G^{\prime} / G\right)$ as follows:

$$
\begin{aligned}
u(\xi)= & \alpha_{-m}\left(\frac{G^{\prime}}{G}\right)^{-m}+\alpha_{-(m-1)}\left(\frac{G^{\prime}}{G}\right)^{-(m-1)}+\cdots \\
& +\alpha_{m-1}\left(\frac{G^{\prime}}{G}\right)^{m-1}+\alpha_{m}\left(\frac{G^{\prime}}{G}\right)^{m}
\end{aligned}
$$

where $G=G(\xi)$ satisfies the second-order linear ordinary differential equation (LODE) in the form

$$
G^{\prime \prime}+\lambda G^{\prime}+\mu G=0,
$$

where $\alpha_{-m}, \ldots, \alpha_{m}, \lambda$, and $\mu$ are constants to be determined later. The unwritten part in (12) is also a polynomial in $\left(G^{\prime} / G\right)$, but the degree of which is generally equal to or less than $m-1$. The positive integer $m$ can be determined by considering the homogeneous balance between the highest-order derivatives and nonlinear terms appearing in (11).

Step 3. Substituting (12) into (11) and using (13), collecting all terms with the same order of $\left(G^{\prime} / G\right)$ together, and then equating each coefficient of the resulting polynomial to zero yields a set of algebraic equations for $\alpha_{m}, \alpha_{m-1}, \ldots, \alpha_{-m}, c, \lambda$, and $\mu$.

Step 4. Since the general solutions of (13) have been well known for us, then substituting $\alpha_{m}, \alpha_{m-1}, \ldots, \alpha_{-m}$ and $c$ and the general solutions of (13) into (12) we have more traveling wave solutions of the nonlinear differential equation (10).

The main idea of $\left(G^{\prime} / G\right)$-expansion method is to use an integrable ODE to expand a solution to a nonlinear partial differential equation (PDE) as a polynomial or rational function of the solution of the ODE. However, such an idea was also presented in [36-38]. The method used in this paper can be also thought of as the application of transformed 
rational function method used in [37] in some sense. Maybe the similar results can be obtained by using these very closely related methods. We plan to further study the EX-ROE in near future by using the methods proposed in [36-38]. We hope we can find much more interesting properties and new phenomenon of this equation.

\section{Exact Traveling Wave Solutions of the Extended Reduced Ostrovsky Equation}

In this section, we will use the $\left(G^{\prime} / G\right)$-expansion method to the extended reduced Ostrovsky equation to get exact traveling wave solutions.

First, in order to get traveling wave solutions, we need some transformation. Recall that in Section 1 we have stated that EX-ROE is equivalent to a Hirota-Satsuma-type shallow water wave equation (7) under the transformation of (8). So here we introduce a new variable $W$ defined by

$$
U=W_{X}
$$

Substituting (14) into (7) yields

$$
W_{X X X T}+p W_{X} W_{X T}+q W_{X X} W_{T}+\beta W_{X T}+q W_{X X}=0
$$

Now giving the traveling wave transformation $W(X, T)=$ $W(\xi), \xi=X-c T$, where $c$ is wave speed. Substituting them into (15) and integrating once, we have

$$
c_{1}+c W_{3 \xi}+\frac{1}{2} c(p+q) W_{\xi}^{2}+(c \beta-q) W_{\xi}=0
$$

where $c_{1}$ is integral constant that is to be determined later.

Considering the homogeneous balance between $W_{3 \xi}$ and $W_{\xi}^{2}$, we have

$$
m+3=2 m+2 \Longrightarrow m=1 \text {. }
$$

We suppose that

$$
W(\xi)=\alpha_{-1}\left(\frac{G^{\prime}}{G}\right)^{-1}+\alpha_{0}+\alpha_{1}\left(\frac{G^{\prime}}{G}\right)
$$

where the $G=G(\xi)$ satisfies the second-order LODE,

$$
G^{\prime \prime}+\lambda G^{\prime}+\mu G=0
$$

and $\alpha_{-1}, \alpha_{0}, \alpha_{1}, \lambda$, and $\mu$ are constants to be determined later.
By using (18) and (19), it is derived that

$$
\begin{aligned}
W_{\xi}= & \mu \alpha_{-1}\left(\frac{G^{\prime}}{G}\right)^{-2}+\lambda \alpha_{-1}\left(\frac{G^{\prime}}{G}\right)^{-1}+\alpha_{-1} \\
& -\alpha_{1} \mu-\lambda \alpha_{1}\left(\frac{G^{\prime}}{G}\right)-\alpha_{1}\left(\frac{G^{\prime}}{G}\right)^{2} \\
W_{\xi}^{2}= & \mu^{2} \alpha_{-1}^{2}\left(\frac{G^{\prime}}{G}\right)^{-4}+2 \lambda \alpha_{-1}^{2} \mu\left(\frac{G^{\prime}}{G}\right)^{-3} \\
& +\left(2 \alpha_{-1}^{2} \mu+\lambda^{2} \alpha_{-1}^{2}-2 \mu \alpha_{-1} \alpha_{-} 1\right)\left(\frac{G^{\prime}}{G}\right)^{-2} \\
& +\left(2 \lambda \alpha_{-1}^{2}-4 \mu \lambda \alpha_{-1} \alpha_{1}\right)\left(\frac{G^{\prime}}{G}\right)^{-1}+\alpha_{-1}^{2}-4 \mu \alpha_{-1} \alpha_{1} \\
& -2 \lambda^{2} \alpha_{-1} \alpha_{1}+\alpha_{1}^{2} \mu^{2}+2 \lambda \alpha_{1}^{2} \mu\left(\frac{G^{\prime}}{G}\right) \\
& +\left(2 \alpha_{1}^{2} \mu+\lambda^{2} \alpha_{1}^{2}\right)\left(\frac{G^{\prime}}{G}\right)^{2}+2 \lambda \alpha_{1}^{2}\left(\frac{G^{\prime}}{G}\right)^{3}+\alpha_{1}^{2}\left(\frac{G^{\prime}}{G}\right)^{4}
\end{aligned}
$$

$$
\begin{aligned}
W_{3 \xi}= & 6 \alpha_{-1} \mu^{3}\left(\frac{G^{\prime}}{G}\right)^{-4}+12 \alpha_{-1} \lambda \mu^{2}\left(\frac{G^{\prime}}{G}\right)^{-3} \\
& -\left(8 \mu^{2} \alpha_{-1}+7 \alpha_{-1} \lambda^{2} \mu\right)\left(\frac{G^{\prime}}{G}\right)^{-2} \\
& -\left(8 \alpha_{-1} \lambda \mu+\alpha_{-1} \lambda^{3}\right)\left(\frac{G^{\prime}}{G}\right)^{-1} \\
& -\left(2 \alpha_{-1} \mu+\lambda^{2} \alpha_{-1}\right)-\left(2 \alpha_{1} \mu^{2}+\lambda^{2} \alpha_{1} \mu\right) \\
& -\left(8 \alpha_{1} \lambda \mu+\alpha_{1} \lambda^{3}\right)\left(\frac{G^{\prime}}{G}\right)-\left(8 \mu \alpha_{1}+7 \alpha_{1} \lambda^{2}\right)\left(\frac{G^{\prime}}{G}\right)^{2} \\
& -12 \alpha_{1} \lambda\left(\frac{G^{\prime}}{G}\right)^{3}-6 \alpha_{1}\left(\frac{G^{\prime}}{G}\right)^{4} .
\end{aligned}
$$

By substituting (20)-(22) into (16) and collecting all terms with the same power of $\left(G^{\prime} / G\right)$ together, the left-hand sides of (16) are converted into the polynomials in $\left(G^{\prime} / G\right)$. Equating the coefficients of the polynomials to zero yields a set of simultaneous algebraic equations for $\alpha_{-1}, \alpha_{0}, \alpha_{1}, \lambda, c, c_{1}$, and $\mu$ as follows (denote $A$ for $\left(G^{\prime} / G\right)$ ):

$$
\begin{aligned}
& A^{-4}: 6 c \alpha_{-1} \mu^{3}+\frac{c(p+q) \mu^{2} \alpha_{-1}^{2}}{2}=0, \\
& A^{-3}: 12 c \lambda \mu^{2} \alpha_{-1}+c(p+q) \lambda \mu \alpha_{-1}^{2}=0,
\end{aligned}
$$


$A^{-2}:(q-c \beta) \mu \alpha_{-1}$

$$
\begin{aligned}
& -\frac{c(p+q)\left(\lambda^{2} \alpha_{-1}^{2}+2 \mu \lambda_{-1}^{2}-2 \mu^{2} \alpha_{1} \alpha_{-1}\right)}{2} \\
& -c\left(7 \lambda^{2} \mu \alpha_{-1}+8 \mu^{2} \alpha_{-1}\right)=0, \\
& A^{-1}:(c \beta-q) \lambda \alpha_{-1} \lambda+c(p+q)\left(\lambda \alpha_{-1}^{2}-2 \lambda \mu \alpha \alpha_{-1}\right) \\
& +c\left(\lambda^{3} \alpha_{-1}+8 \lambda \mu \alpha_{-1}\right)=0, \\
& A^{0}: c_{1}+c\left(\lambda^{2} \alpha_{-1}+2 \mu \alpha_{-1}\right) \\
& -\frac{c\left(2 \alpha_{1} \mu^{2}+\lambda^{2} \alpha_{1} \mu\right)+c(p+q) \alpha_{-1}^{2}}{2} \\
& -2 c \mu \alpha \alpha_{-1}(p+q)+\frac{c \alpha_{1}^{2} \mu^{2}(p+q)}{2}-(c \beta-q) \mu \alpha_{1} \\
& -c \lambda^{2} \alpha_{1} \alpha_{-1}(p+q)+\alpha_{-1}(c \beta-q)=0, \\
& A^{1}:(q-c \beta) \alpha_{1} \lambda+c(p+q)\left(\lambda \mu \alpha_{1}^{2}-2 \lambda \alpha_{1} \alpha_{-1}\right) \\
& -c\left(\lambda^{3} \alpha_{1}+8 \lambda \mu \alpha_{1}\right)=0, \\
& A^{2}:(q-c \beta) \alpha_{1}+c(p+q) \\
& \times\left(\lambda^{2} \alpha_{1}^{2}+2 a \alpha_{1}^{2} \mu-2 \alpha_{1} \alpha_{-1}-c\left(7 \lambda^{2} \alpha_{1}+8 \mu \alpha_{1}\right)\right) \\
& =0 \text {, } \\
& A^{3}:-12 c \lambda \alpha_{1}+c(p+q) \lambda \alpha_{1}^{2}=0, \\
& A^{4}:-6 c \alpha_{1}+\frac{c(p+q) \alpha_{1}^{2}}{2}=0 \text {. }
\end{aligned}
$$

Solving the algebraic equations above yields

$$
\begin{gathered}
\alpha_{1}=\frac{12}{(p+q)}, \\
c=\frac{q}{\left(\beta+\lambda^{2}-4 \mu\right)}, \\
c_{1}=0, \quad \alpha_{-1}=0,
\end{gathered}
$$

or

$$
\begin{aligned}
& \alpha_{-1}=-\frac{12 \mu}{(p+q)}, \\
& c=\frac{q}{\left(\beta+\lambda^{2}-4 \mu\right)}, \\
& c_{1}=0, \quad \alpha_{1}=0 .
\end{aligned}
$$

Substituting system (24) and (25) into (18), we have the formula of the solutions of (15) as follows:

$$
W(X, T)=W(\xi)=\frac{12}{(p+q)}\left(\frac{G^{\prime}}{G}\right)+\alpha_{0},
$$

or

$$
W(X, T)=W(\xi)=-\frac{12 \mu}{(p+q)}\left(\frac{G^{\prime}}{G}\right)^{-1}+\alpha_{0}
$$

where $G$ satisfies (19), $\xi=X-q T /\left(\beta+\lambda^{2}-4 \mu\right)$, and $\alpha_{0}$ is an arbitrary constant.

Since the general solutions $G=G(\xi)$ (hence $G^{\prime}=$ $\mathrm{d} G / \mathrm{d} \xi$ ) of ODE (19) have been known for us, substituting the solutions of (19) into (24) and (25), we have the general traveling wave solutions of (15) as follows.

Case 1. When $\lambda^{2}-4 \mu>0$, then we have the following exact traveling wave solution of (15):

$$
\begin{aligned}
& W_{1}(X, T) \\
& =W_{1}(\xi)=\frac{6 \sqrt{\lambda^{2}-4 \mu}}{p+q} \\
& \times\left(\left(A_{1} \cosh \left(\frac{1}{2} \sqrt{\lambda^{2}-4 \mu \xi}\right)\right)\right. \\
& \left.+A_{2} \sinh \left(\frac{1}{2} \sqrt{\lambda^{2}-4 \mu \xi}\right)\right) \\
& \times\left(A_{1} \sinh \left(\frac{1}{2} \sqrt{\lambda^{2}-4 \mu \xi}\right)\right. \\
& \left.\left.\quad+\frac{6 \lambda}{(p+q)+A_{2}} \cosh \left(\frac{1}{2} \sqrt{\lambda^{2}-4 \mu} \xi\right)\right)^{-1}\right)
\end{aligned}
$$

or

$W_{2}(X, T)$

$$
\begin{aligned}
& =W_{2}(\xi) \\
& =-24 \mu \times((p+q) \\
& \times\left(\sqrt{\lambda^{2}-4 \mu}\right. \\
& \times\left(\left(A_{1} \cosh \left(\frac{1}{2} \sqrt{\lambda^{2}-4 \mu \xi}\right)\right.\right. \\
& \left.\quad+A_{2} \sinh \left(\frac{1}{2} \sqrt{\lambda^{2}-4 \mu \xi}\right)\right) \\
& \times\left(A_{1} \sinh \left(\frac{1}{2} \sqrt{\lambda^{2}-4 \mu} \xi\right)\right. \\
& -\lambda))^{-1}+\alpha_{0},
\end{aligned}
$$


where $\xi=X-q T /\left(\beta+\lambda^{2}-4 \mu\right)$ and $\alpha_{0}, A_{1}, A_{2}$ are arbitrary constants.

Case 2. When $\lambda^{2}-4 \mu<0$, then we have the following exact traveling wave solution of (15):

$W_{3}(X, T)$

$$
\begin{aligned}
&= W_{3}(\xi)=\frac{6 \sqrt{4 \mu-\lambda^{2}}}{p+q} \\
& \times\left(\left(-A_{1} \sin \left(\frac{1}{2} \sqrt{4 \mu-\lambda^{2}} \xi\right)+A_{2} \cos \left(\frac{1}{2} \sqrt{4 \mu-\lambda^{2}} \xi\right)\right)\right. \\
& \quad \times\left(A_{1} \cos \left(\frac{1}{2} \sqrt{4 \mu-\lambda^{2}} \xi\right)\right. \\
&\left.\left.\quad+A_{2} \sin \left(\frac{1}{2} \sqrt{4 \mu-\lambda^{2}} \xi\right)\right)^{-1}\right) \\
&-\frac{6 \lambda}{(p+q)+\alpha_{0}}
\end{aligned}
$$

or

$$
\begin{aligned}
& W_{4}(X, T) \\
& =W_{4}(\xi) \\
& =-24 \mu \\
& \times\left(( p + q ) \left(\sqrt{\lambda^{2}-4 \mu}\right.\right. \\
& \times\left(\left(-A_{1} \sin \left(\frac{1}{2} \sqrt{4 \mu-\lambda^{2}} \xi\right)\right.\right. \\
& \left.+A_{2} \cos \left(\frac{1}{2} \sqrt{4 \mu-\lambda^{2}} \xi\right)\right) \\
& \quad \times\left(A_{1} \cos \left(\frac{1}{2} \sqrt{4 \mu-\lambda^{2}} \xi\right)\right. \\
& \left.\left.+A_{2} \sin \left(\frac{1}{2} \sqrt{4 \mu-\lambda^{2}} \xi\right)\right)^{-1}\right) \\
& \quad-\lambda))^{-1}+\alpha_{0},
\end{aligned}
$$

where $\xi=X-q T /\left(\beta+\lambda^{2}-4 \mu\right)$ and $\alpha_{0}, A_{1}, A_{2}$ are arbitrary constants.

Case 3. When $\lambda^{2}-4 \mu=0$, then we have the following exact rational solution of (15):

$$
W_{5}(X, T)=W_{5}(\xi)=\frac{12}{p+q}\left(\frac{A_{2}}{A_{1}+A_{2} \xi}\right)-\frac{6 \lambda}{(p+q)}+\alpha_{0}
$$

or

$$
W_{6}(X, T)=W_{6}(\xi)=-\frac{24 \mu\left(A_{1}+A_{2} \xi\right)}{(p+q)\left[2 A_{2}-\lambda\left(A_{1}+A_{2} \xi\right)\right]}+\alpha_{0},
$$

where $\xi=X-q T /\left(\beta+\lambda^{2}-4 \mu\right)$ and $\alpha_{0}, A_{1}, A_{2}$ are arbitrary constants.

Now we will show how to get exact traveling wave solutions of (6). From (8) and (14), the solution of EX-ROE (6) is given in parametric form, with $T$ as the parameter, by

$$
u(x, t)=U(t, T), \quad x=\theta(t, T),
$$

where

$$
\theta(X, T)=T+W(X, T)+x_{0} .
$$

So by using (8), (14), (34), (35), (28), and (29), we obtain a parameterized hyperbolic-function-type traveling wave solution of (6) as follows:

$$
\begin{gathered}
u_{1}(x, t) \\
=3\left(A_{2}^{2}-A_{1}^{2}\right)\left(\lambda^{2}-4 \mu\right) \\
\times((p+q) \\
\times\left(A_{1} \sinh \left(\frac{1}{2} \sqrt{\lambda^{2}-4 \mu} \xi\right)\right. \\
\left.\left.+A_{2} \cosh \left(\frac{1}{2} \sqrt{\lambda^{2}-4 \mu} \xi\right)\right)^{2}\right)^{-1}, \\
x=T+W_{1}(t, T)+x_{0}, \\
\text { or } \quad \\
=-12 \mu\left(A_{2}^{2}-A_{1}^{2}\right)\left(\lambda^{2}-4 \mu\right) \\
\times((p+q) \\
\times\left[\left(A_{1} \sqrt{\lambda^{2}-4 \mu}-A_{2} \lambda\right) \cosh \left(\frac{1}{2} \sqrt{\lambda^{2}-4 \mu} \xi\right)\right. \\
+\left(A_{2} \sqrt{\lambda^{2}-4 \mu}-A_{1} \lambda\right) \\
\left.\left.\times \sinh \left(\frac{1}{2} \sqrt{\lambda^{2}-4 \mu} \xi\right)\right]^{2}\right), \\
x=T+W_{2}(t, T)+x_{0},
\end{gathered}
$$

where $\xi=t-q T /\left(\beta+\lambda^{2}-4 \mu\right)$ and $x_{0}$ is an arbitrary constant. 
By using (8), (14), (34), (35), (30), and (31), we obtain a parameterized trigonometric-function-type traveling wave solution of (6) as follows:

$$
\begin{aligned}
& u_{3}(x, t) \\
& =-3\left(A_{2}^{2}+A_{1}^{2}\right)\left(\lambda^{2}-4 \mu\right) \\
& \times((p+q) \\
& \times\left(A_{1} \cos \left(\frac{1}{2} \sqrt{\lambda^{2}-4 \mu \xi}\right)\right. \\
& \left.\left.+A_{2} \sin \left(\frac{1}{2} \sqrt{\lambda^{2}-4 \mu} \xi\right)\right)^{2}\right)^{-1}, \\
& x=T+W_{3}(t, T)+x_{0},
\end{aligned}
$$

or

$$
\begin{aligned}
& u_{4}(x, t) \\
& =-12 \mu\left(A_{2}^{2}-A_{1}^{2}\right)\left(\lambda^{2}-4 \mu\right) \\
& \times((p+q) \\
& \times\left[\left(-A_{2} \sqrt{\lambda^{2}-4 \mu}+A_{1} \lambda\right) \cos \left(\frac{1}{2} \sqrt{\lambda^{2}-4 \mu \xi}\right)\right. \\
& +\left(A_{1} \sqrt{\lambda^{2}-4 \mu}+A_{2} \lambda\right) \\
& \left.\left.\times \sin \left(\frac{1}{2} \sqrt{\lambda^{2}-4 \mu \xi}\right)\right]^{2}\right)^{-1}, \\
& x=T+W_{4}(t, T)+x_{0},
\end{aligned}
$$

where $\xi=t-q T /\left(\beta+\lambda^{2}-4 \mu\right)$ and $x_{0}$ is an arbitrary constant.

By using (8), (14), (34), (35), (32), and (33), we obtain a parameterized rational-type traveling wave solution of (6) as follows:

$$
\begin{gathered}
u_{5}(x, t)=-\frac{12 A_{2}^{2}}{(p+q)\left(A_{1}+A_{2} \xi\right)^{2}}, \\
x=T+W_{5}(t, T)+x_{0},
\end{gathered}
$$

or

$$
\begin{gathered}
u_{6}(x, t)=-\frac{48 \mu A_{2}^{2}}{(p+q)\left(\lambda A_{1}+(-2+\lambda \xi) A_{2}\right)^{2}}, \\
x=T+W_{6}(t, T)+x_{0},
\end{gathered}
$$

where $\xi=t-q T /\left(\beta+\lambda^{2}-4 \mu\right)$ and $x_{0}$ is an arbitrary constant. To our knowledge, these solutions are presented for the first time; they are new exact solutions of EX-ROE.
If we take $A_{1}=0, \mu=0, A_{2} \neq 0$ and $\lambda>0$, then (36) yields the following solitary wave solution of (6):

$$
\begin{gathered}
u(x, t)=\frac{3 \lambda^{2}}{p+q} \operatorname{sech}^{2}\left[\frac{1}{2} \lambda\left(t-\frac{q}{\beta+\lambda^{2}} T\right)\right], \\
x=T+\frac{6 \lambda}{(p+q)} \tanh \left[\frac{1}{2} \lambda\left(t-\frac{q}{\beta+\lambda^{2}} T\right)\right]+x_{0} .
\end{gathered}
$$

Now we will give some discussion of the solitary wave solution (42). Let $\lambda=2 k, x_{0}=0$; the solution (42) is reduced to the solution of (3.26) in [13] after correcting some minor errors [4]. Now from (35), we introduce a new variable:

$$
\chi=x-v t=-v(X-c T)+\frac{6 \lambda}{p+q} \tanh \left[\frac{1}{2} \lambda(X-c T)\right]+x_{0}
$$

where $v=1 / c=\left(\lambda^{2}+\beta\right) / q$. In [8], the authors considered EX-ROE with $p=2 q, \beta \neq 0$ as $\mathrm{mGVE}$ and obtained 1-soliton solution. In fact, if we take $p=2 q, \lambda=2 k$, the solitary wave solution (42) with (43) is reduced to the soliton solution (4.4) and (4.5) in [8]. From the above we can see that the solitary wave solution (3.26) in [4] and the 1-soliton solution of $\mathrm{mGVE}$ are just a special case of the solution (42) in this paper.

\section{Multishaped Solitary Wave Solutions}

In $[8,13]$, the authors showed that the solutions of (4.4) and (4.5), (3.26) and (3.28) may be of different types, namely, loops, cusps, or humps for different values of parameters $\beta$, $k, p$. Here we also show that by choosing different values of the parameters $\beta, \lambda, p, q$, different shape wave solutions can be obtained. As it is stated in Section 1, (9) reduces to VE when $p=q=1, \beta=0$. Taking solution (42) with (43), for example, let $p=q=1, \beta=0, \lambda=2 k$; then it is reduced to one-loop soliton solution (3.4) and (3.5) in [39]. On the other hand, because the solutions of $\mathrm{OHE}$ and VE are connected in a particularly simple way, if we take $p=q=-1, \beta=0, \lambda=2 k$ in (42), we can obtain one-loop soliton solution of OHE.

From above analysis, one can clearly see that the solutions obtained in this paper are generalized for the previous results because here we only take the special case $A_{1}=0, \mu=0$, $A_{2} \neq 0, \lambda>0$ and give special discussion of solution (42). We conclude that if we take different values of the parameters $A_{1}, A_{2}, \lambda, \mu, p, q$, abundancy of types of exact solutions can be obtained from solutions (36), (38), and (40). Here we omit the detailed discussion.

Instead, we give some discussion about solution (37). Science from this solution, multishaped solitary wave solutions can be obtained. Suppose $A_{1} \neq 0, \mu<0, A_{2}=0, \lambda=0$; we reduce solution (37) to

$$
\begin{gathered}
u(x, t)=\frac{12 \mu}{p+q} \operatorname{sech}^{2}\left[\sqrt{-\mu}\left(t-\frac{q}{\beta-4 \mu} T\right)\right], \\
x=T+\frac{24 \sqrt{-\mu}}{(p+q)} \tanh \left[\sqrt{-\mu}\left(t-\frac{q}{\beta-4 \mu} T\right)\right]+x_{0} .
\end{gathered}
$$

We show that for different values of $\beta, \mu$, and $p$, the solution (44) may be of different types. It also owns the property of 


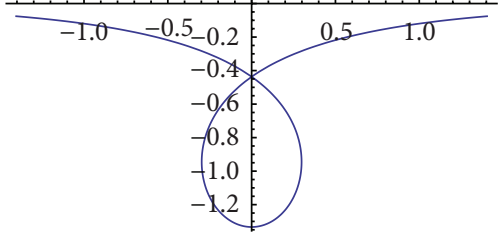

(a)

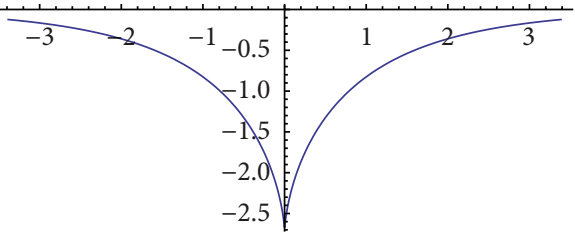

(b)

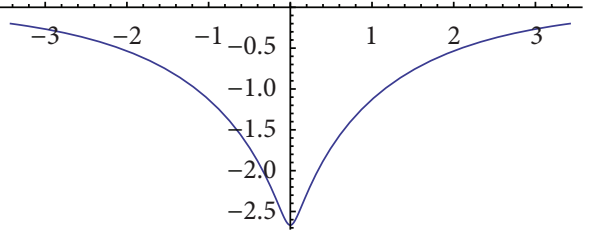

(c)

Figure 1: The profile of solution (44) with $p=3, q=1.5, t=0$, and $x_{0}=0$. For (a) loop-shaped $\beta=0.005$, $\mu=-0.5$, (b) cusp-shaped $\beta=0.05, \mu=-1$, and (c) hump-shaped $\beta=0.5, \mu=-1$.

being loop-shaped, cusp-shaped and hump-shaped, as shown in Figure 1.

\section{Conclusion}

In this paper, we use $\left(G^{\prime} / G\right)$-expansion method to study extended reduced Ostrovsky equation. Several pairs of generalized traveling wave solutions are given directly. These solutions extend the previous results to more general cases. At the same time, multishaped wave solutions can be obtained if the different parameters values are chosen. These explicit solitary wave solutions own the property of being loop-shaped, cusp-shaped, and hump-shaped. These exact traveling wave solutions are also helpful to further study this nonlinear equation which has their physical meaning. The method used in this paper has more advantages. It is direct and concise. Much tedious algebraic calculations can be finished by computer program such as MATHEMATICA and MAPLE. Many well-known nonlinear wave equations can be handled by this method.

\section{Acknowledgment}

The authors thank anonymous referees for valuable suggestions and comments which improve this paper readability and convincibility. This paper is supported by the Starting Research Founding of Wuhan Textile University.

\section{References}

[1] L. A. Ostrovsky, "Nonlinear internal waves in a rotating ocean," Oceanology, vol. 18, pp. 119-125, 1978.

[2] Y. A. Stepanyants, "On stationary solutions of the reduced Ostrovsky equation: periodic waves, compactons and compound solitons," Chaos, Solitons \& Fractals, vol. 28, no. 1, pp. 193-204, 2006.

[3] E. J. Parkes, "Explicit solutions of the reduced Ostrovsky equation," Chaos, Solitons \& Fractals, vol. 31, no. 3, pp. 602-610, 2007.

[4] E. J. Parkes, "Periodic and solitary travelling-wave solutions of an extended reduced Ostrovsky equation," SIGMA, vol. 4, article 053, pp. 1-17, 2008.

[5] J. P. Boyd, "Ostrovsky and Hunter's generic wave equation for weakly dispersive waves: matched asymptotic and pseudospectral study of the paraboloidal travelling waves (corner and nearcorner waves)," European Journal of Applied Mathematics, vol. 16, no. 1, pp. 65-81, 2005.
[6] V. A. Vakhnenko, "Solitons in a nonlinear model medium," Journal of Physics A, vol. 25, no. 15, pp. 4181-4187, 1992.

[7] V. O. Vakhnenko, "High-frequency soliton-like waves in a relaxing medium," Journal of Mathematical Physics, vol. 40, no. 4, pp. 2011-2020, 1999.

[8] A. J. Morrison and E. J. Parkes, “The $N$-soliton solution of the modified generalised Vakhnenko equation (a new nonlinear evolution equation)," Chaos, Solitons \& Fractals, vol. 16, no. 1, pp. 13-26, 2003.

[9] A. Espinosa and J. Fujioka, "Hydrodynamic foundation and Painlevé analysis of Hirota-Satsuma-type equations," Journal of the Physical Society of Japan, vol. 63, no. 4, pp. 1289-1294, 1994.

[10] M. J. Ablowitz, D. J. Kaup, A. C. Newell, and H. Segur, "The inverse scattering transform-Fourier analysis for nonlinear problems," vol. 53, no. 4, pp. 249-315, 1974.

[11] R. Hirota and J. Satsuma, " $N$-soliton solutions of model equations for shallow water waves," vol. 40, no. 2, pp. 611-612, 1976.

[12] A. J. Morrison and E. J. Parkes, "The $N$-soliton solution of a generalised Vakhnenko equation," Glasgow Mathematical Journal, vol. 43A, pp. 65-90, 2001, Integrable systems: linear and nonlinear dynamics (Islay, 1999).

[13] Y. Liu, Z. Li, and K. Wang, "Symbolic computation of exact solutions for a nonlinear evolution equation," Chaos, Solitons \& Fractals, vol. 31, no. 5, pp. 1173-1180, 2007.

[14] S. Xie and J. Cai, "Exact compacton and generalized kink wave solutions of the extended reduced Ostrovsky equation," Communications in Nonlinear Science and Numerical Simulation, vol. 14, no. 9-10, pp. 3561-3573, 2009.

[15] Y. A. Stepanyants, "Solutions classification to the extended reduced Ostrovsky equation," SIGMA, vol. 4, article 073, pp. 119, 2008.

[16] M. Song, "Application of bifurcation method to the generalized Zakharov equations," Abstract and Applied Analysis, vol. 2012, Article ID 308326, 8 pages, 2012.

[17] M. Song, S. A. Bouthina, and A. Biswas, "Topological soliton solution and bifurcation analysis of the Klein-GordonZakharov equation in $(1+1)$-dimensions with power law nonlinearity," Journal of Applied Mathematics, vol. 2013, Article ID 972416, 7 pages, 2013.

[18] A. Biswas and M. Song, "Soliton solution and bifurcation analysis of the Zakharov-Kuznetsov-Benjamin-Bona-Mahoney equation with power law nonlinearity," Communications in Nonlinear Science and Numerical Simulation, vol. 18, pp. 16761683, 2013.

[19] M. Song, C. Yang, and B. Zhang, "Exact solitary wave solutions of the Kadomtsov-Petviashvili-Benjamin-Bona-Mahony equation," Applied Mathematics and Computation, vol. 217, no. 4, pp. 1334-1339, 2010. 
[20] B.-G. Zhang, Z.-R. Liu, and Q. Xiao, "New exact solitary wave and multiple soliton solutions of quantum Zakharov-Kuznetsov equation," Applied Mathematics and Computation, vol. 217, no. 1, pp. 392-402, 2010.

[21] B.-G. Zhang, S.-Y. Li, and Z.-R. Liu, "Homotopy perturbation method for modified Camassa-Holm and Degasperis-Procesi equations," Physics Letters A, vol. 372, no. 11, pp. 1867-1872, 2008.

[22] J. Li and F. Chen, "Exact travelling wave solutions and their dynamical behavior for a class coupled nonlinear wave equations," Discrete and Continuous Dynamical Systems. Series B, vol. 18, no. 1, pp. 163-172, 2013.

[23] J. Li, "Existence of exact families of traveling wave solutions for the sixth-order Ramani equation and a coupled Ramani equation," International Journal of Bifurcation and Chaos in Applied Sciences and Engineering, vol. 22, no. 1, Article ID 1250002, 2 pages, 2012.

[24] C. Pan and Z. Liu, "Further results on the traveling wave solutions for an integrable equation," Journal of Applied Mathematics, Article ID 681383, 6 pages, 2013.

[25] Z. Liu and Y. Liang, "The explicit nonlinear wave solutions and their bifurcations of the generalized Camassa-Holm equation," International Journal of Bifurcation and Chaos in Applied Sciences and Engineering, vol. 21, no. 11, pp. 3119-3136, 2011.

[26] H.-T. Chen, S.-H. Yang, and W.-X. Ma, "Double sub-equation method for complexiton solutions of nonlinear partial differential equations," Applied Mathematics and Computation, vol. 219, no. 9, pp. 4775-4781, 2013.

[27] W.-X. Ma and Y. Liu, "Invariant subspaces and exact solutions of a class of dispersive evolution equations," Communications in Nonlinear Science and Numerical Simulation, vol. 17, no. 10, pp. 3795-3801, 2012.

[28] M. Wang, X. Li, and J. Zhang, "The $\left(G^{\prime} / G\right)$-expansion method and travelling wave solutions of nonlinear evolution equations in mathematical physics," Physics Letters A, vol. 372, no. 4, pp. 417-423, 2008.

[29] M. Wang, J. Zhang, and X. Li, "Application of the $\left(G^{\prime} / G\right)$-expansion to travelling wave solutions of the Broer-Kaup and the approximate long water wave equations," Applied Mathematics and Computation, vol. 206, no. 1, pp. 321-326, 2008.

[30] D. D. Ganji and M. Abdollahzadeh, "Exact traveling solutions of some nonlinear evolution equation by $\left(G^{\prime} / G\right)$-expansion method," Journal of Mathematical Physics, vol. 50, no. 1, Article ID 013519, p. 10, 2009.

[31] Y.-B. Zhou and C. Li, "Application of modified $G^{\prime} / G$-expansion method to traveling wave solutions for Whitham-Broer-Kauplike equations," Communications in Theoretical Physics, vol. 51, no. 4, pp. 664-670, 2009.

[32] H. Zhang, "New application of the $\left(G^{\prime} / G\right)$-expansion method," Communications in Nonlinear Science and Numerical Simulation, vol. 14, pp. 3220-3225, 2009.

[33] S. Zhang, J. L. Tong, and W. Wang, "A generalized $\left(G^{\prime} / G\right)$ expansion method for the $\mathrm{mKdV}$ equation with variable coefficients," Physics Letters A, vol. 372, pp. 2254-2257, 2008.

[34] J. Zhang, X. Wei, and Y. Lu, "A generalized $\left(G^{\prime} / G\right)$-expansion method and its applications," Physics Letters A, vol. 372, no. 20, pp. 3653-3658, 2008.

[35] W. A. Li, H. Chen, and G. C. Zhang, "The $(\omega / g)$-expansion method and its application to Vakhnenko equation," Chinese Physics B, vol. 18, pp. 400-405, 2009.

[36] W. X. Ma and B. Fuchssteiner, "Explicit and exact solutions to a Kolmogorov-Petrovskii-Piskunov equation," International Journal of Non-Linear Mechanics, vol. 31, no. 3, pp. 329-338, 1996.
[37] W.-X. Ma and J.-H. Lee, "A transformed rational function method and exact solutions to the $3+1$ dimensional JimboMiwa equation," Chaos, Solitons \& Fractals, vol. 42, no. 3, pp. 1356-1363, 2009.

[38] W.-X. Ma and Z. Zhu, "Solving the $(3+1)$-dimensional generalized KP and BKP equations by the multiple exp-function algorithm," Applied Mathematics and Computation, vol. 218, no. 24, pp. 11871-11879, 2012.

[39] V. O. Vakhnenko and E. J. Parkes, "The two loop soliton solution of the Vakhnenko equation," Nonlinearity, vol. 11, no. 6, pp. 1457-1464, 1998. 


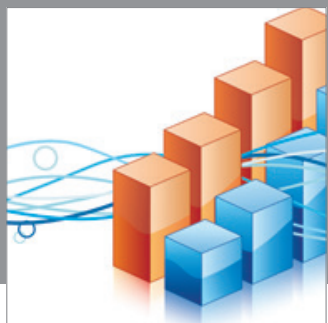

Advances in

Operations Research

mansans

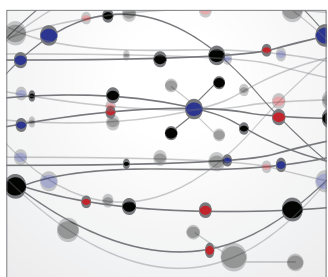

The Scientific World Journal
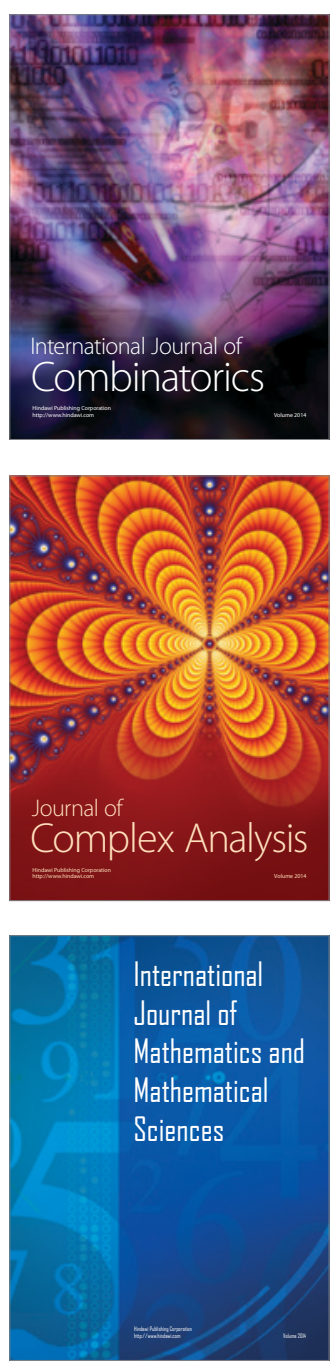
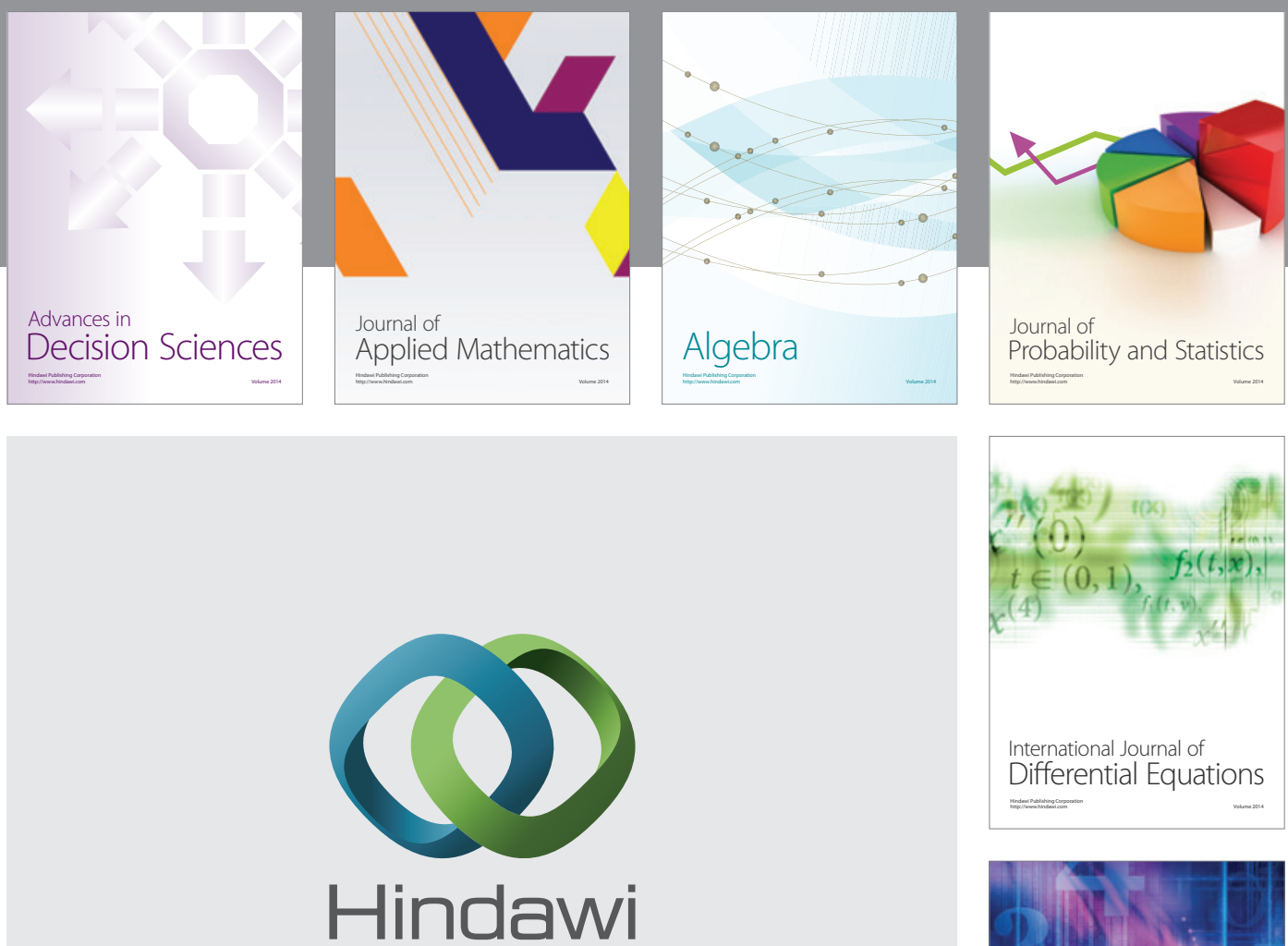

Submit your manuscripts at http://www.hindawi.com
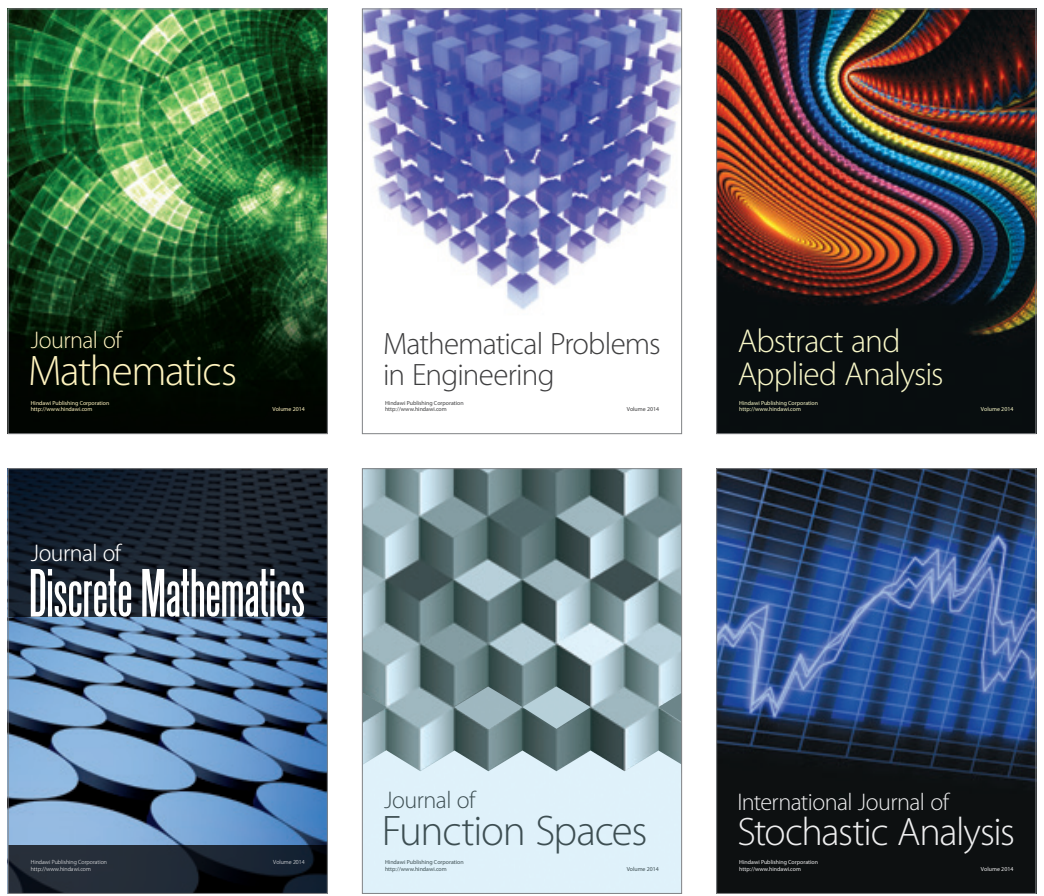

Journal of

Function Spaces

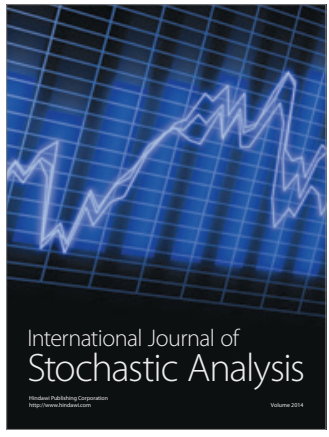

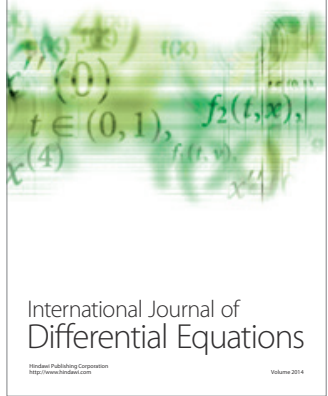
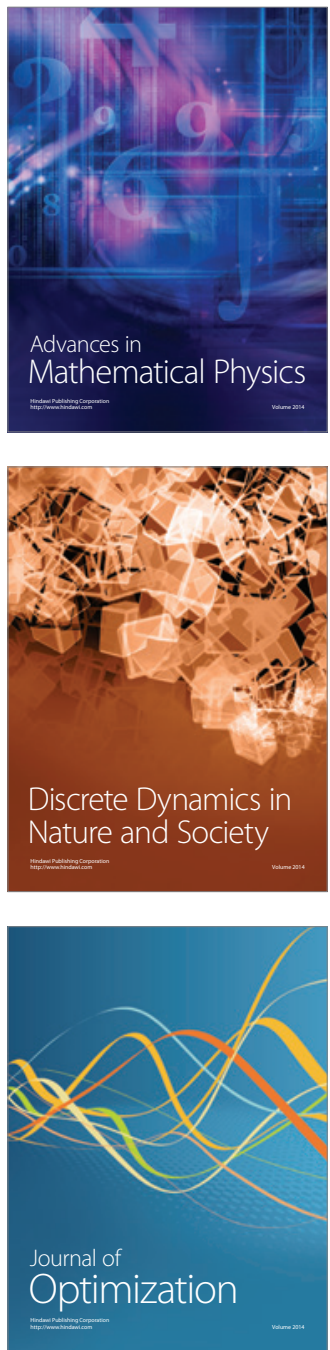